\title{
ARTICLE
}

\section{Development of radionuclide distribution database and map system on the Fukushima nuclear accident}

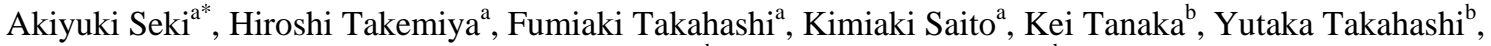 \\ Kazuhiro Takemura ${ }^{\mathrm{b}}$ and Masaharu Tsuzawa ${ }^{\mathrm{b}}$

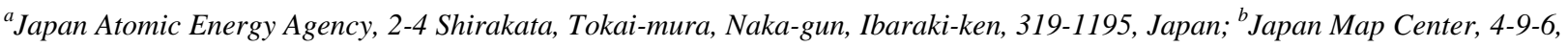 \\ Aobadai, Meguro-Ku, Tokyo-to, 153-8522, Japan
}

\begin{abstract}
The Radionuclide distribution database and map system, which provide basic information for evaluations and countermeasures of the accident at Fukushima Daiichi nuclear power plant, are explained. Due to massive earthquake and tsunami, Fukushima Daiichi nuclear power plant had been damaged and spread out radioactive materials around the Fukushima region. In order to meet the various requirements from government, local government, residents, and/or researchers, we developed two systems to provide those data to the public. One of the systems is a database system which is designed to provide quantitative data for detailed analysis. Another is a map system which provides intuitive images for the qualitative estimation. In the two systems, it is possible to provide the information requested by a wide range of people.
\end{abstract}

Keywords: Fukushima Daiichi nuclear power plant; nuclear accident; air-dose; radionuclide; distribution; database; map; soil sample; car-borne survey; air-borne survey

\section{Introduction}

Many people, such as researchers, officers, and the general publics wanted the information about the distribution of radionuclide around the Fukushima region. For example, there were demands to see the distribution of radiation dose in a residential area, or to use them for making plans of decontamination. To meet the demands, the environmental radioactivity data should be provided to the public in the ways that are easy for them to use. Therefore, the distribution database and map systems [1, 2] were developed. The database system provides the numerical data on the air dose rate and radioactive concentration. Moreover, detailed information such as measurement methods, analysis methods, precision of the measured data and so on, are also provided. While the distribution map system provides visualized information on the distribution of the dose rate in air and radioactive concentration in the soil. The map system opened to the public at the web site of Japan Atomic Energy Agency since October 2011. The database system will be open at a later date.

In this paper, the background of this study is explained first, reviewing the Tohoku earthquake. Then, the monitoring measurements are explained. The distribution database and map systems which we developed for the research are then shown. Finally, the summary of this paper and the future plans are

*Corresponding author. Email: seki.akiyuki@jaea.go.jp explained.

\section{Background}

The Tohoku earthquake happened on March 11, 2011. It was 9.0 magnitudes at the Pacific Ocean from $100 \mathrm{~km}$ of northeastern coast and that scale of earthquake seldom happened in Japan. It generated tsunami which reached about $15 \mathrm{~m}$ at the Fukushima Daiichi nuclear power plant. It broke the power supply for cooling systems in the plant and the nuclear accident happened there.

Along with that, the radionuclides were released and spread throughout the wide region. At the early phase following the accident, the airborne survey was conducted by the Department of Energy (DOE) in USA from March to April, 2011. We got the result that the majority of radioactive plume went to the northwestern part from the nuclear plant. After that, the plume changed its direction to southwestern to the middle part of Fukushima prefecture.

In order to estimate the impact of the nuclear accident and to take appropriate countermeasures, MEXT commenced a project to produce radiation distribution maps under the 2011 Strategic funds for the Promotion of Science and Technology on June 6, 2011. For this project [3], JAEA and other institutes started various kind of radiation monitoring, from which radiation data were acquired for radiation maps. In addition, a database 
system including radiation data was developed by JAEA.

Prior to the preparation of the database and maps, the Review Meeting for the Preparation of Distribution Map of Radiation Dose, etc. was held to discuss technical matter and validity of the result.

\section{Radiation monitoring and mapping}

The various kind of monitoring had been conducted in the project. Especially, the soil sampling, car-borne, air-borne survey results were displayed on the map. The soil sampling survey was required to determine the radionuclides in soil. The car-borne survey was effective in the continuous measurement of the air dose rate. As for the wide region survey, the air-borne survey results were also implemented.

\subsection{Soil sampling survey}

The Figure 1 shows the soil sampling area. In the beginning of this project, we divided the $2 \mathrm{~km}^{2}$ and 10 $\mathrm{km}^{2}$ regions to collect 5 samples in each region. $2 \mathrm{~km}^{2}$ regions are in about $80 \mathrm{~km}$ radius from Fukushima Daiichi nuclear power plant and $10 \mathrm{~km}^{2}$ regions are over $80 \mathrm{~km}$ radius. The numbers of soil samples were greater than 10,000 which were collected by more than 400 people. We used special instrument for collecting the sample from the $5 \mathrm{~cm}$ surface layer. The soil was mixed in a plastic bag for even distribution and packed in the U8 plastic container for detection. After that, the concentrations of nuclide in those soil samples were measured by the Japan Chemical Analysis Center, the University of Tokyo, and other 19 organizations.

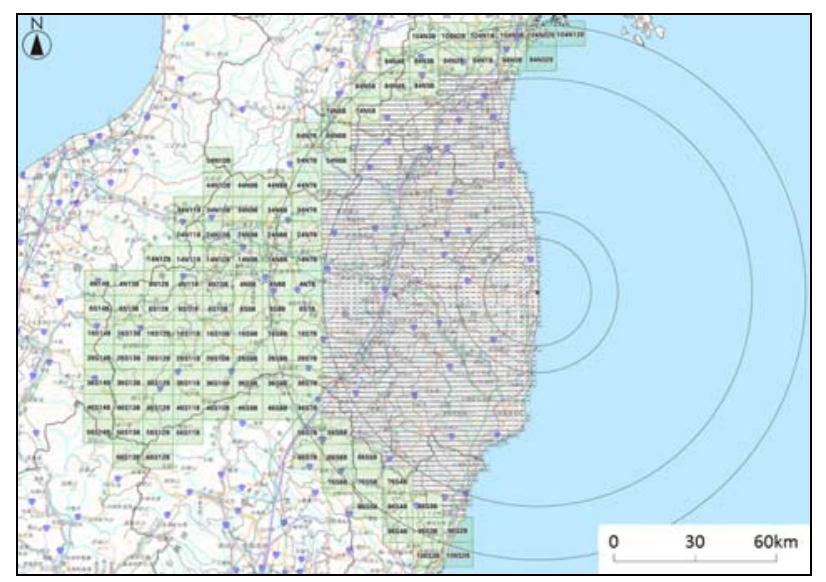

Figure 1. The soil sampling area.

\subsection{Car-borne survey}

The Figure 2 shows the car-borne survey area. The green lines represent the roads which would be surveyed. We used the KURAMA system for the car-borne survey. That system measured the dose rates and GPS data at the same time. And those data were transferred to the storage server through the cellular network. KURAMA system was set in a car. The dose rate was converted to the rate outside because radiation dose was shielded by car. And for precise evaluation to the human body, the dose rate was also adjusted to the height of $1 \mathrm{~m}$ from the ground.

Eventually, the total mileage became more than $17,000 \mathrm{~km}$ but only covered the national and prefectural roads mainly within the Fukushima prefecture. Then, we have started next car-borne survey last December 2011 which commenced not only in Fukushima prefecture but also neighboring prefectures jointly with municipalities. The number of sampling points increased up to 140,000.

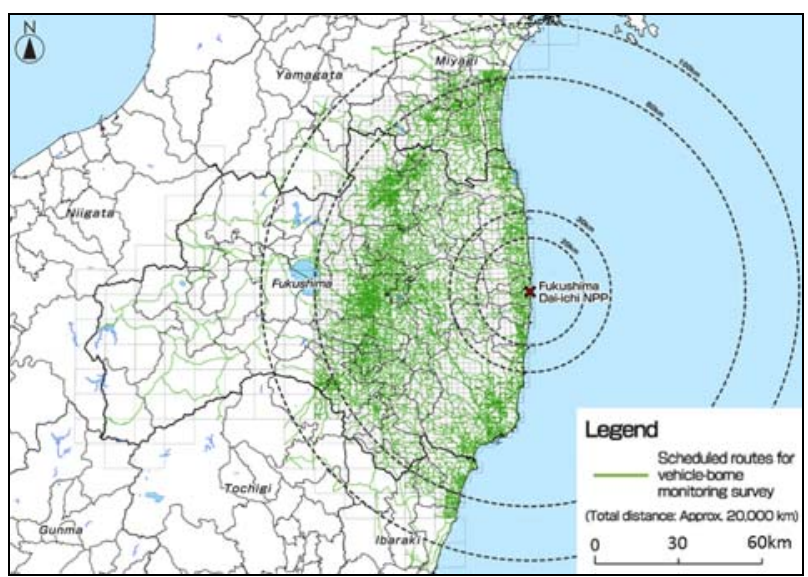

Figure 2. The car-borne survey area.

\subsection{Air-borne survey}

The air-borne survey was done under another research project conducted by MEXT. Those data have been also installed in database and map systems. Like car-borne survey, the amount of gamma-ray spectrum and GPS data were measured for every second by the helicopter. The aerial data were needed to be converted to those on the ground using data from in situ spectrometry on the ground. The survey covered the eastern region of Japan. The number of sampling points became more than 1,400,000.

\section{Development of the infrastructure for the project}

Those monitoring data became enormous amount because those data were measured over wide region in the eastern part of Japan. Furthermore various kinds of measurements were used for promoting the accuracy of environmental information. Those data have been required to open to the public. Finally, those data should be used by different kinds of people. To meet those requirements, the infrastructures were developed for correcting, analyzing and providing those data. The distribution database and map systems were developed for providing data. Figure $\mathbf{3}$ shows images of relationship of those infrastructures.

Before providing data to the public, access loads of both systems were evaluated through stress tests. It was found that the map system suffered from connection failure owing to heavy loads, because this system was designed to create a distribution map on demand 
according to user requests. To solve this problem, we redesigned the system to provide maps prepared in advance. Subsequently, the number of failures was reduced from $20 \%$ to $0.000001 \%$. The map system was accessed by more than 300,000 users over the first 10 days with no failures.

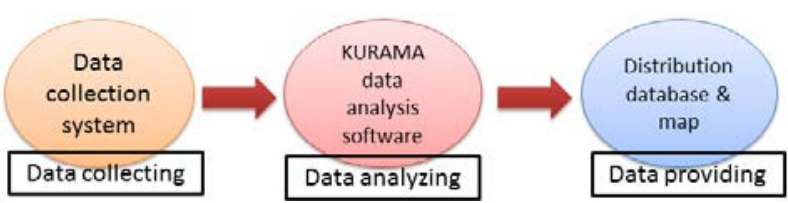

Figure 3. The image of the relationship of the infrastructures which are developed in this project.

\subsection{Distribution database system}

The distribution database system which is available on the internet in a few days has been developed to indicate data on the map in detail. A user can select the type of data, prefecture and municipality, then the data are displayed in the tabulate form, as shown in Figure 4. Furthermore some detailed information such as soil's weight, the type of survey meter can be confirmed in the table. The data can be downloaded with the form of XML files and CSV files from the same web site. Then, the downloaded data can be useful for our works and researches.

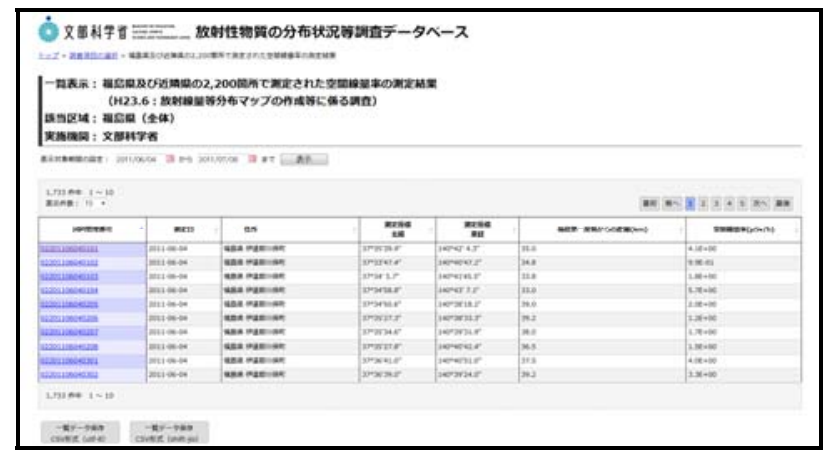

Figure 4. One example of the distribution database system.

\subsection{Distribution map system}

The distribution map system was also developed for providing radiation data to the public. Those maps have been available through the internet since October in 2011. A user can recognize the dose rate in air (unit: Sievert per hour, Sv/h) and contamination of radioactivity in the soil (unit: Becquerel per square meter, $\mathrm{Bq} / \mathrm{m}^{2}$ ) around the place of interest. There are DENSHIKOKUDO, PDF, and smartphone types of maps. DENSHIKOKUDO is the web-based map available on the Digital Japan Portal Web Site. The soil, car-borne, and air-borne survey data are available like Figure 5. The functions of comparison of those data are also available. Moreover DENSHIKOKUD and smartphone types of maps have the zooming-up function which shows dose rate at many locations in detail. And the map has information of places where elementary and junior high schools are located. The functions for searching the name of places or the latitude and the longitude are also available.

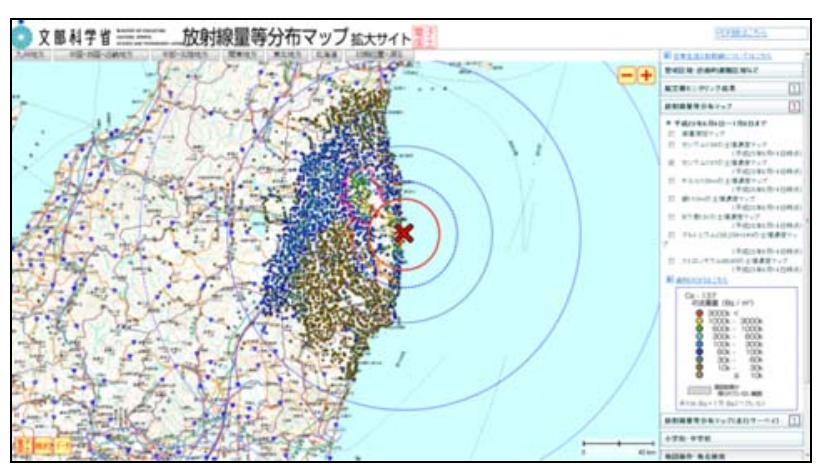

Figure 5. One example of the distribution map system.

\section{Summary and future plans}

The first mapping project has been finished by November 2011. The large amounts of data about the distribution of radionuclide were collected. The distribution database and map systems are developed for providing those data in the ways that are easy for many people to use. Examples of installed data are shown in Table 1.

In the future, the periodical surveys on contamination are continued. Actually, the second mapping project has started since December 2011. Moreover, there are other environmental data which were collected by MEXT, Fukushima prefecture and other organizations. We have a plan to install those data to the same database and map systems. We hope that those systems would be used for evaluations and countermeasures.

Table 1. This table shows the environmental radioactivity data that have been installed by September 2012 .

\begin{tabular}{|c|c|c|}
\hline Installed data & Map & Database \\
\hline $\begin{array}{l}\text { Concentration of Radionuclide } \\
\text { in Soil Samples and Air Dose } \\
\text { Rate at around 2,200 } \\
\text { Locations within } \\
\text { Approximately } 100 \mathrm{~km} \text { from } \\
\text { the Fukushima Dai-ichi NPP. }\end{array}$ & installed & installed \\
\hline $\begin{array}{l}\text { Car-borne Monitoring Survey } \\
\text { within Approximately } 100 \mathrm{~km} \\
\text { from the Fukushima Dai-ichi } \\
\text { NPP }\end{array}$ & installed & nil \\
\hline $\begin{array}{l}\text { Results of Air-borne } \\
\text { Monitoring Survey by MEXT } \\
\text { and DOE }\end{array}$ & installed & nil \\
\hline $\begin{array}{l}\text { Survey on the Movement of } \\
\text { Radioactive Substances in } \\
\text { River Water and Well Water }\end{array}$ & nil & installed \\
\hline $\begin{array}{l}\text { Results of Present Depth } \\
\text { Analysis of Cs Radioactivity at } \\
77 \text { Locations }\end{array}$ & nil & installed \\
\hline
\end{tabular}




\section{Acknowledgements}

I want to thank the Visible Information Center, Inc. for constructing the database system and operating the database and map systems.

\section{References}

[1] The Ministry of Education, Culture, Sports, Science and Technology, Database on the Research of Radioactive Substances Distribution, http://radb.jaea.go.jp/mapdb/. [in Japanese]

[2] The Ministry of Education, Culture, Sports, Science and Technology, Extension Site of Distribution Map of Radiation Dose, etc., http://ramap.jaea.go.jp/map/. [in Japanese]

[3]The Ministry of Education, Culture, Sports, Science and Technology, and the Ministry of Agriculture, Forestry and Fisheries, The study on distribution of radioactive substances around Fukushima Daiichi Nuclear Power Plant, The Report of the FY2011 Strategic Funds for the Promotion of S\&T (2012), pp.1-97 1-104. [in Japanese 